\title{
Probing quantum geometry at LHC
}

\author{
Gia Dvali, ${ }^{a, b, c, d}$ Cesar Gomez $^{e}$ and Slava Mukhanov ${ }^{a, b, d}$ \\ ${ }^{a}$ Arnold Sommerfeld Center for Theoretical Physics, \\ Department für Physik, Ludwig-Maximilians-Universität München, \\ Theresienstr. 37, 80333 München, Germany \\ ${ }^{b}$ Max-Planck-Institut für Physik \\ Föhringer Ring 6, 80805 München, Germany \\ ${ }^{c}$ CERN, Theory Division, \\ 1211 Geneva 23, Switzerland \\ ${ }^{d}$ CCPP, Department of Physics, New York University, \\ 4 Washington Place, New York, NY 10003, U.S.A. \\ e Instituto de Física Teórica UAM-CSIC, C-XVI, \\ Universidad Autónoma de Madrid, Cantoblanco, 28049 Madrid, Spain \\ E-mail: georgi.dvali@cern.ch, bfkl08@gmail.com, \\ Viatcheslav.Mukhanov@physik. uni-muenchen.de
}

ABSTRACT: We present an evidence, that the volumes of compactified spaces as well as the areas of black hole horizons must be quantized in Planck units. This quantization has phenomenological consequences, most dramatic being for micro black holes in the theories with $\mathrm{TeV}$ scale gravity that can be produced at LHC. We predict that black holes come in form of a discrete tower with well defined spacing. Instead of thermal evaporation, they decay through the sequence of spontaneous particle emissions, with each transition reducing the horizon area by strictly integer number of Planck units. Quantization of the horizons can be a crucial missing link by which the notion of the minimal length in gravity eliminates physical singularities. In case when the remnants of the black holes with the minimal possible area and mass of order few $\mathrm{TeV}$ are stable, they might be good candidates for the cold dark matter in the Universe.

Keywords: Large Extra Dimensions, Black Holes

ARXIV EPRINT: 1006.2466 


\section{Contents}

1 Introduction 1

2 Quantum geometry in gravity with extra dimensions $\quad 2$

3 Predictions 5

$\begin{array}{lll}4 & \text { Conclusions and speculations } & 7\end{array}$

\section{Introduction}

The black hole physics dictates the existence of the minimal possible measurable scales for the area and the volume. In fact, in any experiment, which is supposed to measure area (cross section) smaller than the Planck area $L_{P l}^{2} \simeq 10^{-66} \mathrm{~cm}^{2}$ one has to involve particle scattering with the higher than Planckian momentum-transfer, which will form a black hole with the radius exceeding the Planck length $L_{P l}$. Impossibility to probe subPlanckian length-scales suggests that Einstein gravity viewed as field theory is ultra-violet self-complete, but in a sense profoundly different from the conventional (Wilsonian) notion $[1,2]$. The key ingredient is a crossover between the quantum degrees of freedom and classical states (Black holes). This phenomenon among other things implies, that the smallest black holes, with mass $\sim L_{P l}^{-1}$, become sharp quantum resonances, which instead of conventional thermal evaporation are either stable or decay into the light fields in a single quantum jump.

These two facts, impossibility of resolving distances shorter than $L_{P L}$ and crossover of classical black holes into the quantum resonances, suggest that quantum jumps should not be property of exclusively smallest black holes. Rather, black holes must obey some fundamental quantization rule which for a large black hole should reproduce the conventional thermal picture, but should simultaneously explain the gradual transition of the smaller and smaller black holes into the quantum particles.

We shall argue, that the right quantization condition is that the area must be quantized in terms of the integers of the Planck area. In particular, this refers to the well defined area of the black hole horizon. The above assumption is well supported by the fact that the black hole horizon area behaves as adiabatic invariant [3] and by statistical interpretation of the entropy of the quantized black hole [4].

Moreover, we wish to show, that from the requirement that black hole saturate the holographic bound on the information storage [6-13] it follows that in any elementary emission its horizon area cannot be reduced by less than one Planck unit. In fact, let us arrange a "gedanken experiment" in which we tag the information bit without altering the classical black hole geometry. One can achieve this as follows. Assume that we have a spectator light particle that carries a charge under a gauged $Z_{2}$-symmetry, and consider 
black hole evaporation in such a theory. For example, one can prepare a large classical black hole and endow it with a $Z_{2}$-charge by throwing a $Z_{2}$-charged particle in it. Since $Z_{2}$-charge is not associated with any massless electric field, there exist no classical hair under it [14-21], and the effects of quantum hair [22-25] on the classical geometry can be safely ignored. The black hole will then evaporate as the usual neutral Schwarzschild black hole would do. Now consider an elementary act of emission of $Z_{2}$-charged particle. Due to the absence of hair, emission of this particle is not any different from the emission of any other quantum, but the advantage is that the $Z_{2}$-charge allows us to trace the information in a clean way. There is a full single bit of information encoded in this charge. Therefore it is obvious that after particle emission, the horizon area cannot shrink by less than one Planck unit, because otherwise the remaining surface would violate the requirement of the saturation of holographic bound. Likewise, we could arrive to an inconsistency in the inverse process of absorption. ${ }^{1}$

Thus, we see that the only way to maintain holographic bound to be saturated is that elementary jump of the horizon area in any one-particle emission process must be quantized in Planck units, in accordance with the conjecture of [3-5]. The essence of our argument is to transform the abstract concept of information into a measurable $Z_{2}$-charge with no classical-hair.

The area quantization can have a dramatic consequences for the Hawking evaporation of the black holes, especially when it refers to the microscopic black holes $[4,5]$. The quantization of the geometry of the black hole horizon become especially interesting in the theories with large extra dimensions, where the fundamental Planck scale can be lowered by many orders of magnitude and become accessible at LHC.

\section{Quantum geometry in gravity with extra dimensions}

In the theories with extra-dimensions gravity can become $D=4+d$ dimensional on submillimeter or smaller scales and the corresponding quantum gravity Planck scale can be lowed to the scale reachable at LHC $[26,27]$. In fact, assuming that the fundamental theory is a $D$-dimensional Einstein gravity, let us consider compactification of $D$-dimensional spacetime to the direct product of 4-dimensional Minkowski space-time and of a $d$-dimensional compact torus of volume $V_{d}$. Then, it follows from ${ }^{2}$

$$
\frac{1}{L_{D}^{2+d}} \int R \sqrt{-g} d^{4+d} x=\frac{V_{d}}{L_{D}^{2+d}} \int R \sqrt{-g} d^{4} x=\frac{1}{L_{P l}^{2}} \int R \sqrt{-g} d^{4} x
$$

\footnotetext{
${ }^{1}$ Had we instead used either a single particle with a $Z_{N}$-charge or a single member of $N$ distinct particle species family, the information would change by $N$ bits, changing the black hole area by $N L_{P l}^{2}$. This fully

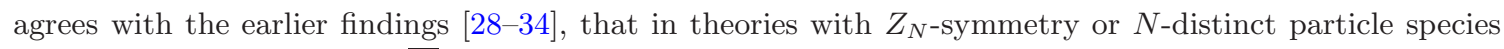
the fundamental length is $\sqrt{N} L_{P l}$, and the area of small black holes (for which gravity is modified ) could instead be quantized in these units. However, due to modification of mass-to-radius relation this gives exactly the same result as quantization of the effective area measured by massless graviton in units of $L_{P l}^{2}$ ! Below we shall witness this connection on explicit example of Kaluza-Klein theories, which propagate tower of modes.

${ }^{2}$ To simplify the formulae we are skipping the numerical coefficients such as $4 \pi$ etc., which reader can easily restore.
} 
that the corresponding 4-dimensional and $D$ - dimensional Planck lengths, denoted by $L_{P l}$ and $L_{D}$ respectively, are related as

$$
L_{D}=\left(V_{d} / L_{D}^{d}\right)^{\frac{1}{2}} L_{P l} .
$$

The the ratio

$$
\left(V_{d} / L_{D}^{d}\right)=N
$$

counts the total number of Kaluza-Klein modes with masses not exceeding $L_{D}^{-1}[28-31,33$, 34]. More massive species should not be counted since they form black holes.

We wish to suggest that the quantization of the black hole horizon area implies quantization of the compactification volume. We shall not prove this rigorously, but only try to provide some qualitative arguments. First, the inverse radius sets the masses of KaluzaKlein states. If we extrapolate this relation to heavy states, and take into the account the fact that at sufficiently high masses particles must cross-over to classical black holes, then the quantization of the latter masses, implies quantization of the compactification radii. Of course, we understand limitations of this argument, since it relies on extrapolating the mass-to-radius relation to the region of trans-Planckian masses, where one can argue that this relation may receive order-one corrections. Nevertheless, it is still informative, since such quantization would look reasonable from the point of view of continuous transition between the heavy particles and the black holes, since it would automatically offer an unified geometric reason to black hole quantization as well as to the heavy-particle/ black hole complementarity.

A related argument can be illustrated on a simple case of $D=5$. Let the compactification volume be $V_{1} \equiv R$ and let us assume $R / L_{5}=N+\epsilon$, where $\epsilon<1$. If this were possible, then we could choose $N=0$, meaning that $R=\epsilon L_{5}$. That is, we could take the compactification radius arbitrarily smaller than $L_{5}$. But in this case the fifth dimension stops existence in any physical sense. In particular, $L_{5}$ becomes shorter than the four-dimensional Planck length, $L_{P l}$, and the would-be KK modes become ultra-Planckian mass states and are indistinguishable from four-dimensional classical black holes, according to $[1,2]$. The extra dimension ceases to exist. In other words, quantization of compact volume automatically follows if $R$ is forced to be bigger than $L_{5}$ and this should be the case due to the fact that trans-Planckian states are equivalent to classical black holes.

For $N \simeq 10^{32}$ the $D$-dimensional Planck length $L_{D}$ becomes of order $L_{D} \simeq 10^{-17} \mathrm{~cm}$ and corresponds to $D$-dimensional nonperturbative quantum gravity scale reachable at LHC. Moreover, from the relation between the mass and gravitational radius,

$$
M_{B H}=\frac{R_{g}^{1+d}}{L_{D}^{2+d}},
$$

it follows that the minimal black hole with $R_{g} \simeq L_{D}$ has mass $M_{B H} \simeq L_{D}^{-1} \simeq O$ (1) Tev and hence such black holes can be produced at LHC. They should be described by a nonperturbative $D$-dimensional quantum gravity which can substantially revise the process of their Hawking evaporation. In fact, below we will show that the assumption about the area quantization leads to a dramatic modification of the Hawking's result, obtained in the approximation of the external classical gravitational field. 
In $D$-dimensional quantum gravity the area of the $D$-dimensional Schwarzschild black hole should satisfy the following quantization rule

$$
A_{D}=n L_{D}^{D-2} \text {. }
$$

This means, that an effective projection of the horizon area $A_{D}$, measured by a $D$ dimensional observer, must be equal to the integer number of the corresponding $D$ dimensional Planck units.

One may wonder in which units the area should be quantized from the point of view of 4-dimensional observer when $d=D-4$ dimensions are compactified on the torus of volume $V_{d} \equiv R^{d}$, where $R$ is the compactification radius. Let us show that in this case the quantization rule (2.4) implies that the 4-dimensional projection of the the black hole area is automatically quantized in terms of $4 \mathrm{~d}$ Planck units $L_{P l}^{2} \simeq 10^{-66} \mathrm{~cm}^{2}$. This is a manifestation of a general phenomenon that the Bekenstein-Hawking entropies computed for one and the same black hole by $D$-dimensional and $D-d$-dimensional observers in their respective Planck units, exactly match [35].

We consider first a black hole of radius $R_{g} \gg R$. The horizon of such black hole has a geometry of $S_{2} \times T_{d}$, where $S_{2}$ is an usual 4-dimensional Schwarzschild two-sphere of area $A_{4}=R_{g}^{2}$, and $T_{d}$ is a $d$-dimensional torus of volume $V_{d}=R^{d}$; hence, $A_{D}=A_{4} V_{d}$. According to (2.4) the $2+d$-dimensional horizon area should be quantized in units of $L_{D}^{2+d}$ and we obtain

$$
n=A_{D} / L_{D}^{2+d}=R_{g}^{2} V_{d} / L_{D}^{2+d}=A_{4} / L_{P l}^{2},
$$

where we have taken into account relation (2.2). Thus, we have shown, that the projection of the horizon of a $D$-dimensional black hole in $4 d$ space-time consists of as many $4 d$ Planck areas $L_{P l}^{2}$ as the original $D-2$ dimensional horizon contains corresponding Planck units $L_{D}^{2+d}$.

For the black hole of size $R_{g} \ll R$, the matching of two quantizations conditions is a little bit more subtle. The geometry of such a black hole to a very good approximation is given by $D$-dimensional Schwarzschild geometry, with the horizon being a $2+d$ dimensional sphere of area $A_{D}=R_{g}^{2+d}$. However, a four-dimensional observer only measures a 4-dimensional projection of this area, which is a two-dimensional sphere of the effective area $A_{4}=R_{g}^{2}\left(R_{g}^{d} / V_{d}\right)$. The additional factor $\left(R_{g}^{d} / V_{d}\right)$ is due to the suppression of the overlap of the KK wave-function profile in $T_{d}$ with the area of the BH. Quantizing this area in units of four-dimensional Planck area $L_{P l}^{2}$, we get,

$$
n=A_{4} / L_{P l}^{2}=R_{g}^{2}\left(R_{g}^{d} / V_{d}\right) L_{P l}^{-2},
$$

or, using the relation $(2.2)$, we can rewrite this as,

$$
n=A_{D} / L_{D}^{2+d} \text {. }
$$

The latter expression is a quantization of the area in units $L_{D}^{2+d}$ with exactly the same $n$ !

This is a remarkable result, which means that the entropy of the quantized black hole, which according to [4] is equal to

$$
S=(n-1) \ln 2,
$$

is the same from the points of view of $D$-dimensional and 4-dimensional observers and it is proportional to the respective areas measured in the corresponding Planck units. 


\section{Predictions}

There are two obvious places where one could look for the experimental signatures of our results. These are micro black holes, that may be accessible at LHC, and cosmology.

Not surprisingly, most dramatic phenomenological impact the horizon area quantization has on the microscopic black holes of about $\mathrm{TeV}$ mass, which are predicted in the scenario with large extra dimensions [26, 27]. Let us focus on the case when the size of the black holes, that can be potentially produced at LHC, is much smaller than the compactification radius and hence the effects of compactification can be ignored.

The mass of the Schwarzschild black hole in $D=4+d$ dimensions can be written in terms of the horizon area $A$ as,

$$
M=M_{D}^{2+d} A^{\frac{1+d}{2+d}}
$$

where $M_{D}=L_{D}^{-1}$ is the minimal possible mass for the black hole. The quantization condition (2.4) implies that the mass is also quantized,

$$
M_{n}=M_{D} n^{\frac{1+d}{2+d}}
$$

and if the distance between levels is larger than the width of the levels the black hole evaporation has to be considered as the sequence of discrete transitions. The elementary transition between two nearby levels corresponds to the change of the area by one Plank unit $L_{D}^{d+2}$. As a result of such transition the black hole emits a particle of the energy

$$
\varepsilon_{n}=M_{n}-M_{n-1}=M_{D}\left(n^{\frac{1+d}{2+d}}-(n-1)^{\frac{1+d}{2+d}}\right) .
$$

For $n \gg 1$ this can be approximated by

$$
\varepsilon_{n} \simeq \frac{1+d}{2+d} R_{g}^{-1}
$$

For a large black hole, with $R_{g} \gg L_{D}$, the direct transitions from level $n$ to $n-2$ and other nearby levels reproduce the thermal properties of spectrum of the radiation emitted from the "box" of finite size $R_{g}$ with an effective Hawking temperature $T \equiv R_{g}^{-1}[5]$. However, the crucial difference is that the large wavelengths exceeding $R_{g}$ are not present for an arbitrarily large black hole. For small black holes, that are of LHC interest, the difference is even more dramatic. Rather than viewed as evaporation, the decay process of a small black hole represents a sequence of spontaneous emissions with the total number of emitted quanta given by

$$
n_{\text {total }} \simeq\left(M_{B H} L_{D}\right)^{\frac{2+d}{1+d}} .
$$

We shall now estimate the emission rate and the lifetime of the black without relying on the thermal properties of radiation.

Let us consider the decay via graviton emission, treating it as a sequence of the spontaneous emissions. Since each step is a quantum emission process with a momentum transfer 
$\varepsilon_{n}$, by general covariance [36] the graviton emission rate per unit of the Planck area, corresponding to such momentum-transfer is,

$$
\tilde{\Gamma}_{n}=\alpha\left(L_{D} \varepsilon_{n}\right)^{2+d} \varepsilon_{n}=\alpha M_{D}\left(n^{\frac{1+d}{2+d}}-(n-1)^{\frac{1+d}{2+d}}\right)^{3+d}
$$

where $\alpha$ is some numerical coefficient which seems to be significantly smaller than unity because of the existence of the external classical barrier [5]. ${ }^{3}$ To get the total emission rate we have to multiply (3.6) by the areas in the Planck units and hence the total emission rate is

$$
\Gamma_{n}=\tilde{\Gamma}_{n}\left(\frac{R_{g}^{2+d}}{L_{D}^{2+d}}\right)=n \tilde{\Gamma}_{n} .
$$

This expression describes the rate of transition $n \rightarrow n-1$. Taking into account (3.4) for large $n$ it can be approximated as

$$
\Gamma_{n} \simeq \alpha \varepsilon_{n}
$$

Because $\alpha$ seems to be much smaller than unity even with taking into account the emission of other particles [5] the width of the levels, proportional to $\Gamma_{n}$, is much smaller than the distance between the levels $\varepsilon_{n}$. Therefore one expects that small black hole will emit particles in rather sharp lines corresponding to the energy difference $\varepsilon_{n}$. The characteristic time of the transition with one particle emission is given by,

$$
\tau_{n} \simeq \Gamma_{n}^{-1}=n^{-1} \tilde{\Gamma}_{n}^{-1} .
$$

For a large black hole, $n \gg 1$, the half life-time, corresponding to the emission of about $n$ quanta, obtained using approximations (3.4), (3.8), is

$$
T_{n} \simeq n \tau_{n} \sim \alpha^{-1} n^{\frac{3+d}{2+d}} L_{D} \sim \alpha^{-1} R_{g}^{3+d} M_{D}^{2+d} .
$$

For small black holes one has to use more precise expressions (3.3), (3.6) and (3.7) instead. These expressions indicate that a small black hole essentially decays as a quantum particle. This is in agreement with the finding of [1,2], that Einstein gravity must propagate quantum degrees of freedom with mass of order $M_{D}$, which can be viewed as the very end points of black hole evaporation. The above results fully support this picture. Thus as anticipated, even if LHC reaches the threshold of the black formation, the first accessible quantum-gravity states will be indistinguishable from the particles.

The maximal values of $n$ that can be probed at center of mass energy $E_{c}$, is given by $n=\left(E_{c} / M_{D}\right)^{\frac{d+2}{d+1}}$, and the momenta of the first decay products soften according to the following law,

$$
p=M_{D}\left(\left(E_{c} / M_{D}\right)-\left(\left(E_{c} / M_{D}\right)^{\frac{d+2}{d+1}}-1\right)^{\frac{1+d}{2+d}}\right) .
$$

This softening is the characteristic signal of the beginning of the black hole production at LHC, but its detection will requires reaching higher values of $n$.

\footnotetext{
${ }^{3}$ Note that due to smallness of $\alpha$ the smallest black holes are sharp quantum resonances in accordance with findings of $[33,34]$
} 


\section{Conclusions and speculations}

We have argued that the existence of the minimal length in gravity, when combined with black hole no-hair properties and holographic bound on information storage, implies quantization of geometric areas in Planck units. We have discussed some fundamental and phenomenological implications of this quantization. Below we shall briefly summarize these implications as well as some future prospects for the quantized geometry.

Black hole tower at LHC. A most dramatic impact quantization of the black hole horizon has for $\mathrm{TeV}$ mass black holes that are predicted in theories with low Planck mass scenarios $[26-31]{ }^{4}$ As shown above, in pure gravity such black holes will come in form of the quantum resonances spaced according to (3.3). In realistic scenario one also has to include Standard Model particle localized on a 3-brane. As shown in [33, 34], their presence increases the minimal length to $L_{N}=N_{S M}^{\frac{1}{d+2}} L_{D}$, where $N_{S M}$ is the number of Standard model helicities per helicity of a $4+d$-dimensional graviton. The black hole horizon now must be quantized in $L_{N}$-units, but due to a small number of Standard Model species and the suppression by the exponential factor $1 /(d+2)$, sensitivity in the change is inessential,

$$
M_{n}=\left(\frac{M_{D}}{N_{S M}^{\frac{1}{d+2}}}\right) n^{\frac{1+d}{2+d}} .
$$

Already for $d=2$ this renormalizes the quantization unit only by factor of 2 . So the influence of the Standard Model particles on the black hole quantization can be ignored.

The above quantization implies that the properties of the micro black holes are nothing like the conventional view of semi-classical thermal states. Instead, they will come as sharp quantum resonances, with characteristic trajectory given by (3.2).

Micro black holes can have essentially any quantum number that can be composed out of particle states in the Standard Model, and thus, can imitate many weakly coupled extensions of the Standard Model, such as, extra gauge-bosons, supersymmetry, or other exotic models. Thus, in certain sense gravity projects the spectrum of all possible extensions of the Standard Model at the fixed scale around $M_{D}$. The only way to recognize the black hole nature of these new states, is to create highly excited black holes that should obey the spacing (3.3).

In particular, if, as suggested in $[33,34]$, the scattering of longitudinal $W^{ \pm}$-bosons gets unitarized by black holes, the "fake" Higgs particle will be a lowest excitation of one such tower.

The interesting phenomenological question would be to differentiate the black hole tower from a more conventional string Regge trajectory, also predicted in [27] (for more detailed phenomenological studies of this effect see, [38-44]). Such a tower, will open up first, if the Standard Model is UV-completed by a weakly-coupled string theory, but will be replaced by the black hole tower if UV-completion happens by gravity (or if string theory is strongly coupled).

\footnotetext{
${ }^{4}$ Note by V.M.: if it is confirmed then Gia will buy me $10^{3}$ bottles of wine, as opposed to $10^{32}$ particles that these theories propagate.
} 
Implication for cosmological singularities. The area-quantization can be a crucial missing link in reconciling the notion of the minimal length, that is responsible for selfcompleteness of gravity, with cosmological singularities.

Indeed, if the Hubble volume is quantized in $L_{P l}$-units, the final states of the collapsing universe may be similar to the black hole evaporation. As we have argued above, a black hole with $n=1$ will decay into a single quantum jump into the lighter states. Similarly, the collapsing Universe after reaching the Planckian density will represent collection of Hubble patches each of the area $n=1$. Despite the fact that number of patches may be enormous, each patch can only undergo a discrete quantum transition which changes $n$ by an integer. A transition from $n=1$ to $n=0$ corresponds to the decay of a given patch into nothingness. Of course, this "decay into nothingness" requires to be given a precise meaning. Since the purpose of our discussion is not to address these subtleties, but rather set possible future directions for thinking, we can only say, that this process can be thought as an inverse of creation out of nothing [45-48]. In an Universe where the latter process is allowed and has a physical meaning, so must be the inverse one. Since the patches are causally disconnected, we expect that the transitions are not correlated. In this way, the total volume of the Universe is reduced by an integer Planck volumes, without further increase of density. The Hubble patches with $n=1$ decay into nothingness within a single quantum jump, without passing through singularity.

Similarly, Universe can be created out of nothing as a result of a quantum transition [45-48], but since $n$ can change only by an integer in any Hubble patch, passing through a classical singularity is avoided. This concepts require further study that will not be attempted here.

Implication for moduli. Quantization of compactification volume implies quantization on the vacuum expectation values of the moduli fields. In this way moduli cannot assume arbitrary values, and this may shed some new light on issues of moduli stabilization and perhaps on supersymmetry breaking. Consequently, the values of the coupling constants that are set by moduli fields should also obey certain quantization rule. In the same spirit, applying quantization of areas in Planck units to string theory and taking into the account the fact that string length must play the role of the fundamental scale, we conclude that string coupling, $1 / g_{s}^{2}$, should also be quantized. This is in agreement with the results of $[36,37]$ according to which $1 / g_{s}^{2}$ measures the effective number of particles species to which the semi-classical black holes can decay normalized to the rate of emission of graviton helicities.

Implication for time-dependent backgrounds. The other important application of our results is for cosmological time-dependent backgrounds, in which horizons and volumes change, provided one speculates and extends the quantization of black hole horizon to the quantization of cosmological horizons and or compactification volumes. Then, in particular, it follows that change of expectation values of the scalar fields in the early universe cannot be continuous, but rather has to proceed via quantum jumps, each of which changes compactification or Hubble volume by one Planck unit. This follows from the fact that in inflationary cosmology the Hubble volume is set by the value of the scalar potential, 
determined by the expectation value of the scalar field. In the conventional approach the latter can change continuously resulting into a continuous change of Hubble parameter. But if we impose quantization on the latter, this would imply that the scalar field can no longer assume arbitrary values. This discretization of motion is especially important for small compactification volumes and horizons, and could have imprint in early cosmology, especially when one considers the self reproducing eternal universe.

Implications for cold dark matter. The geometry quantization may also have an interesting cosmological applications for Dark Matter. It might happen that the minimal black holes of mass $M_{D} \simeq O(1)$ Tev (corresponding to $n=1$ ) are stable. Then they have about right geometrical cross section to be good candidate for the cold dark matter in our universe. In other words, their geometric cross-section being $\sim 1 /(\mathrm{TeV})^{2}$ is comparable to standard WIMP cross-section (which assuming initial thermal distribution) gives a right abundance. Of course, cosmology of such dark matter candidate has to be reconsidered in the light of strong gravity effects and other factors that are not present in the standard WIPM scenario. This analysis is beyond the scope of the present work.

\section{Acknowledgments}

The work of G.D. was supported in part by Humboldt Foundation under Alexander von Humboldt Professorship, by European Commission under the ERC advanced grant 226371, by David and Lucile Packard Foundation Fellowship for Science and Engineering and by the NSF grant PHY-0758032. The work of C.G. was supported in part by Grants: FPA 2009-07908, CPAN (CSD2007-00042) and HEPHACOS P-ESP00346. V.M. is supported by TRR 33 "The Dark Universe" and the Cluster of Excellence EXC 153 "Origin and Structure of the Universe".

Open Access. This article is distributed under the terms of the Creative Commons Attribution Noncommercial License which permits any noncommercial use, distribution, and reproduction in any medium, provided the original author(s) and source are credited.

\section{References}

[1] G. Dvali and C. Gomez, Self-completeness of Einstein gravity, arXiv:1005.3497 [SPIRES].

[2] G. Dvali, S. Folkerts and C. Germani, Physics of Trans-Planckian Gravity, arXiv: 1006.0984 [SPIRES].

[3] J.D. Bekenstein, The quantum mass spectrum of the Kerr black hole, Lett. Nuovo Cim. 11 (1974) 467.

[4] V. Mukhanov, Are black holes quantized?, Pis. Eksp. Teor. Fiz. 44 (1986) 50 [JETP Lett. 44 (1986) 63].

[5] J.D. Bekenstein and V.F. Mukhanov, Spectroscopy of the quantum black hole, Phys. Lett. B 360 (1995) 7 [gr-qc/9505012] [SPIRES].

[6] J.D. Bekenstein, A universal upper bound on the entropy to energy ratio for bounded systems, Phys. Rev. D 23 (1981) 287 [SPIRES]. 
[7] W.G. Unruh and R.M. Wald, Acceleration radiation and generalized second law of thermodynamics, Phys. Rev. D 25 (1982) 942 [SPIRES].

[8] W.G. Unruh and R.M. Wald, Entropy bounds, acceleration radiation, and the generalized second law, Phys. Rev. D 27 (1983) 2271.

[9] J.D. Bekenstein, Specific entropy and the sign of the energy, Phys. Rev. D 26 (1982) 950 [SPIRES].

[10] J.D. Bekenstein, Entropy bounds and black hole remnants, Phys. Rev. D 49 (1994) 1912 [gr-qc/9307035] [SPIRES].

[11] G. 't Hooft, Dimensional reduction in quantum gravity, gr-qc/9310026 [SPIRES].

[12] L. Susskind, The World as a hologram, J. Math. Phys. 36 (1995) 6377 [hep-th/9409089] [SPIRES].

[13] L. Susskind and E. Witten, The holographic bound in Anti-de Sitter space, hep-th/9805114 [SPIRES].

[14] W. Israel, Event horizons in static vacuum space-times, Phys. Rev. 164 (1967) 1776 [SPIRES].

[15] W. Israel, Event horizon in static electrovac space-times, Commun. Math. Phys. 8 (1968) 245.

[16] B. Carter, Axisymmetric black hole has only two degrees of freedom, Phys. Rev. Lett. 26 (1971) 331 [SPIRES].

[17] J.B. Hartle, Long-range neutrino forces exerted by Kerr black holes, Phys. Rev. D 3 (1971) 2938 [SPIRES].

[18] J.D. Bekenstein, Nonexistence of baryon number for static black holes, Phys. Rev. D 5 (1972) 1239 [SPIRES].

[19] J.D. Bekenstein, Nonexistence of baryon number for black holes. II, Phys. Rev. D 5 (1972) 2403 [SPIRES].

[20] J.D. Bekenstein, Transcendence of the law of baryon-number conservation in black hole physics, Phys. Rev. Lett. 28 (1972) 452 [SPIRES].

[21] C. Teitelboim, Nonmeasurability of the quantum numbers of a black hole, Phys. Rev. D 5 (1972) 2941.

[22] L.M. Krauss and F. Wilczek, Discrete gauge symmetry in continuum theories, Phys. Rev. Lett. 62 (1989) 1221 [SPIRES].

[23] S.R. Coleman, J. Preskill and F. Wilczek, Dynamical effect of quantum hair, Mod. Phys. Lett. A 6 (1991) 1631 [SPIRES].

[24] S.R. Coleman, J. Preskill and F. Wilczek, Growing hair on black holes, Phys. Rev. Lett. 67 (1991) 1975 [SPIRES].

[25] S.R. Coleman, J. Preskill and F. Wilczek, Quantum hair on black holes, Nucl. Phys. B 378 (1992) 175 [hep-th/9201059] [SPIRES].

[26] N. Arkani-Hamed, S. Dimopoulos and G.R. Dvali, The hierarchy problem and new dimensions at a millimeter, Phys. Lett. B 429 (1998) 263 [hep-ph/9803315] [SPIRES].

[27] I. Antoniadis, N. Arkani-Hamed, S. Dimopoulos and G.R. Dvali, New dimensions at a millimeter to a Fermi and superstrings at a TeV, Phys. Lett. B 436 (1998) 257 [hep-ph/9804398] [SPIRES]. 
[28] G. Dvali, Black holes and large- $N$ species solution to the hierarchy problem, Fortsch. Phys. 58 (2010) 528 [arXiv: 0706 . 2050] [SPIRES].

[29] G. Dvali and M. Redi, Black hole bound on the number of species and quantum gravity at LHC, Phys. Rev. D 77 (2008) 045027 [arXiv: 0710.4344] [SPIRES].

[30] G. Dvali and M. Redi, Phenomenology of $10^{3} 2$ dark sectors, Phys. Rev. D 80 (2009) 055001 [arXiv:0905.1709] [SPIRES].

[31] R. Brustein, G. Dvali and G. Veneziano, A bound on the effective gravitational coupling from semiclassical black holes, JHEP 10 (2009) 085 [arXiv:0907.5516] [SPIRES].

[32] G. Dvali, M. Redi, S. Sibiryakov and A. Vainshtein, Gravity cutoff in theories with large discrete symmetries, Phys. Rev. Lett. 101 (2008) 151603 [arXiv:0804.0769] [SPIRES].

[33] G. Dvali and C. Gomez, Quantum information and gravity cutoff in theories with species, Phys. Lett. B 674 (2009) 303 [arXiv:0812.1940] [SPIRES].

[34] G. Dvali and C. Gomez, Strong coupling holography, arXiv:0907.3237 [SPIRES].

[35] G. Dvali and S.N. Solodukhin, Black hole entropy and gravity cutoff, arXiv:0806.3976 [SPIRES].

[36] G. Dvali and D. Lüst, Evaporation of microscopic black holes in string theory and the bound on species, Fortsch. Phys. 58 (2010) 505 [arXiv:0912.3167] [SPIRES].

[37] G. Dvali and C. Gomez, Species and strings, arXiv:1004.3744 [SPIRES].

[38] S. Cullen, M. Perelstein and M.E. Peskin, TeV strings and collider probes of large extra dimensions, Phys. Rev. D 62 (2000) 055012 [hep-ph/0001166] [SPIRES].

[39] D. Lüst, S. Stieberger and T.R. Taylor, The LHC string hunter's companion, Nucl. Phys. B 808 (2009) 1 [arXiv:0807.3333] [SPIRES].

[40] L.A. Anchordoqui et al., Dijet signals for low mass strings at the LHC, Phys. Rev. Lett. 101 (2008) 241803 [arXiv:0808. 0497] [SPIRES].

[41] L.A. Anchordoqui et al., LHC phenomenology for string hunters, Nucl. Phys. B 821 (2009) 181 [arXiv:0904.3547] [SPIRES].

[42] D. Lüst, O. Schlotterer, S. Stieberger and T.R. Taylor, The LHC string hunter's companion (II): five-particle amplitudes and universal properties, Nucl. Phys. B 828 (2010) 139 [arXiv: 0908.0409] [SPIRES].

[43] P. Burikham, T. Figy and T. Han, TeV-scale string resonances at hadron colliders, Phys. Rev. D 71 (2005) 016005 [hep-ph/0411094] [SPIRES].

[44] Z. Dong, T. Han, M.-x. Huang and G. Shiu, Top quarks as a window to string resonances, JHEP 09 (2010) 048 [arXiv: 1004.5441] [SPIRES].

[45] L.P. Grishchuk and Ya.B. Zeldovich, Complete cosmological theories, in Quantum structure of space and time, M. Duff and C. Isham eds., Cambridge University Press, Cambridge U.K. (1983).

[46] A. Vilenkin, Creation of universes from nothing, Phys. Lett. B 117 (1982) 25 [SPIRES].

[47] J.B. Hartle and S.W. Hawking, Wave function of the universe, Phys. Rev. D 28 (1983) 2960 [SPIRES].

[48] A.D. Linde, Quantum creation of the inflationary universe, Lett. Nuovo Cim. 39 (1984) 401. 